\title{
Computer Vision-Based Assessment of Motor Functioning in Schizophrenia: Use of Smartphones for Remote Measurement of Schizophrenia Symptomatology
}

\author{
Anzar Abbas ${ }^{a}$ Vijay Yadav ${ }^{a} \quad$ Emma Smith $^{b}$ Elizabeth Ramjas ${ }^{b}$ \\ Sarah B. Rutter ${ }^{b}$ Caridad Benavidez ${ }^{b}$ Vidya Koesmahargyo ${ }^{a}$ \\ Li Zhang $^{\text {a }}$ Lei Guan $^{\mathrm{a}}$ Paul Rosenfield ${ }^{\mathrm{b}}$ Mercedes Perez-Rodriguez ${ }^{\mathrm{b}}$ \\ Isaac R. Galatzer-Levya, c \\ ${ }^{a}$ AiCure, LLC, New York, NY, USA; ${ }^{b}$ Icahn School of Medicine at Mount Sinai, New York, NY, USA; \\ 'Psychiatry, New York University School of Medicine, New York, NY, USA
}

\author{
Keywords \\ Schizophrenia $\cdot$ Head movement $\cdot$ Motor function · Digital \\ phenotyping
}

\begin{abstract}
Introduction: Motor abnormalities have been shown to be a distinct component of schizophrenia symptomatology. However, objective and scalable methods for assessment of motor functioning in schizophrenia are lacking. Advancements in machine learning-based digital tools have allowed for automated and remote "digital phenotyping" of disease symptomatology. Here, we assess the performance of a computer vision-based assessment of motor functioning as a characteristic of schizophrenia using video data collected remotely through smartphones. Methods: Eighteen patients with schizophrenia and 9 healthy controls were asked to remotely participate in smartphone-based assessments daily for 14 days. Video recorded from the smartphone front-facing camera during these assessments was used to quantify the Euclidean distance of head movement between frames
\end{abstract}

through a pretrained computer vision model. The ability of head movement measurements to distinguish between patients and healthy controls as well as their relationship to schizophrenia symptom severity as measured through traditional clinical scores was assessed. Results: The rate of head movement in participants with schizophrenia $(1.48 \mathrm{~mm} /$ frame) and those without differed significantly $(2.50 \mathrm{~mm} /$ frame; $p=0.01$ ), and a logistic regression demonstrated that head movement was a significant predictor of schizophrenia diagnosis $(p=0.02)$. Linear regression between head movement and clinical scores of schizophrenia showed that head movement has a negative relationship with schizophrenia symptom severity $(p=0.04)$, primarily with negative symptoms of schizophrenia. Conclusions: Remote, smartphonebased assessments were able to capture meaningful visual behavior for computer vision-based objective measurement of head movement. The measurements of head movement acquired were able to accurately classify schizophrenia diagnosis and quantify symptom severity in patients with schizophrenia.

(c) 2021 The Author(s)

Published by S. Karger AG, Basel karger@karger.com www.karger.com/dib

Karger $\stackrel{\text { '⿳亠丷厂 }}{ }$

BOPEN ACCESS
(C) 2021 The Author(s)

Published by S. Karger AG, Basel

This is an Open Access article licensed under the Creative Commons Attribution-NonCommercial-4.0 International License (CC BY-NC) (http://www.karger.com/Services/OpenAccessLicense), applicable to the online version of the article only. Usage and distribution for commercial purposes requires written permission.
Vidya Koesmahargyo

AiCure, LLC

19 W 24th Street

New York, NY 11375 (USA)

vidya.koesmahargyo@aicure.com 


\section{Introduction}

Motor abnormalities have been shown to be a characteristic trait of schizophrenia, are present even in antipsychotic-naive patients, predate the onset of psychosis, and have been demonstrated in at-risk populations and in unaffected relatives [1-6]. Indeed, psychomotor retardation underlies core negative symptomatology of schizophrenia, such as the demonstration of blunted facial affect and emotional withdrawal [7-11]. Though there have been many attempts to diagnose schizophrenia and quantify symptom severity using neurobiology and behavior [1214], little work has been done to objectively measure motor dysfunction to characterize the disorder in clinical research [15-17], which could be of particular relevance in the assessment of negative symptoms.

Recent advances in the mechanistic understanding of negative symptomatology have led to a number of promising pharmacological and cognitive treatments for negative symptoms of schizophrenia [18-21]. Such initiatives are important given the lack of FDA-approved treatments for negative symptoms [22]. However, reliable and change-sensitive measures of negative symptomatology to assess the efficacy of these treatments are sparse [2326]. With motor dysfunction being an underlying factor of negative symptomatology, accurate measurement of motor functioning can allow for assessment of treatment efficacy during the evaluation of novel investigational treatments.

Several groups have successfully and accurately measured motor functioning as a characteristic of schizophrenia, though only in laboratory settings [27-31]. While laboratory-based methodologies increase sensitivity and reduce subjectivity in the measurement of motor functioning compared to traditional assessments, they are limited in their utility as measurement tools in clinical research given the burden imposed on both patients and clinicians.

The use of "digital phenotyping" or measurement of disease symptomatology using digital tools has shown significant promise towards objective, scalable, and remote measurement of central nervous system functioning $[32,33]$. In schizophrenia, observable behavior associated with the disorder has been successfully quantified using advancements in machine learning. Examples of this include using digital signal and natural language processing methods to measure changes in verbal prosody and speech characteristics, such as volume, fundamental frequency, pause characteristics, and sentiment, and computer vision to quantify changes in facial ex- pressivity and the presence of blunted facial affect, a core negative symptom of schizophrenia [34-38]. The use of visual data such as videos of patient behavior presents a promising avenue for digital phenotyping given the ubiquitous availability of smartphone cameras alongside advances in computer vision methodologies that can automate the processing and analysis of visual information. Despite recent advances in head pose estimation $[39,40]$, there are a lack of studies utilizing these methods to quantify head movement abnormalities in schizophrenia.

In the current investigation, we examined the ability of head movement measured using computer vision from videos recorded during remote smartphone-based assessments to differentiate individuals with schizophrenia from healthy controls and determine disease severity by comparison of head movement with the Positive and Negative Syndrome Scale (PANSS), a commonly used clinical assessment tool for the measurement of schizophrenia severity and the current "gold standard" for assessment of antipsychotic treatment efficacy [41].

\section{Methods}

\section{Participants}

Patients meeting DSM-5 criteria for schizophrenia at Mount Sinai Health System Outpatient Psychiatry Clinics $(n=18$; age $\mu=$ 48.1 years, $\sigma=13.1 ; 11$ females) and healthy individuals from the community ( $n=9$; age $\mu=39.8$ years, $\sigma=10.4$; 5 females) were consented to participate in a 2 -week observational study under approval of the Mount Sinai Program for the Protection of Human Subjects and its Institutional Review Board. Participants in the schizophrenia group were on a stable regimen of psychotropic medications, had no recent clinically meaningful change in schizophrenia symptomatology, and were clinically stable in that they were not expected to show significant changes in symptomatology over the course of the 2-week observational study.

\section{Data Collection}

Positive and Negative Syndrome Scale (PANSS)

The PANSS was administered in-person by the study team on both patients with schizophrenia and healthy control subjects. Healthy individuals were assessed to confirm an absence of schizophrenia symptomatology. From the PANSS, subscale scores were recorded for the positive symptom subscale (P Total), the negative symptom subscale (N Total), and general symptom subscale (G Total) in addition to all individual items within those subscales.

Remote Smartphone-Based Video Assessments

All participants were asked to download the AiCure app (www. aicure.com) on either their personal smartphone or a smartphone provided to them by the study team. They were then trained by the study team on how to use the app to participate in remote assessments. The remote assessments triggered the participants to per- 
form a brief and standardized 1-min task where they were asked open-ended questions to which they responded freely while a video of their response was captured using the front-facing camera on the smartphone. The open-ended questions were neutral in nature and simply meant to elicit a free verbal response (e.g., "What have you been doing for the past few hours?" and "What are your plans for the rest of the day?"). All participants were shown the same prompts and stimuli to ensure standardization and reproducibility of the manner in which all data were collected. Dependent on the length of the participant's responses, the videos collected would have been of different lengths, hence introducing variability. However, all observations reported in this manuscript reflect average movement behavior over the course of those videos, and hence the length of the video is not a factor in any analyses. The assessments were scheduled every day for the 14 days of the study. The videos collected during these assessments were used to quantify head movement during the participants' responses.

\section{Measurements of Head Movement}

The software library OpenFace [42-44] was used to measure framewise head movement from the videos collected through the remote smartphone-based assessments. For each frame, the head's position relative to the camera was calculated using pretrained convolutional neural network-based computer vision models. The software provides a confidence score for every frame of video denoting the likelihood that it is accurately detecting a face; only frames with a confidence score of $80 \%$ or higher were used for downstream analyses. Framewise measurements of change in Euclidean head position in the $x, y$, and $z$ planes were calculated in millimeters as shown in the equation below. From the framewise measurements, the mean head movement was calculated across all videos collected from a participant over the course of the study. All measurements of head movement were normalized between 0 and 1 before subsequent data analysis. Head movement in frame:

$$
n=\sqrt{\left(x_{n}-x_{n-1}\right)^{2}+\left(y_{n}-y_{n-1}\right)^{2}+\left(z_{n}-z_{n-1}\right)^{2}},
$$

where

$x_{n}=$ head position in the $x$ plane during the current frame,

$y_{n}=$ head position in the $y$ plane during the current frame, and

$z_{n}=$ head position in the $z$ plane during the current frame.

\section{Data Analysis}

Using head movement data collected from all participants in both groups, a multiple logistic regression with maximum likelihood estimation was conducted to model the probability of an individual having a schizophrenia diagnosis based on measurements of head movement, with age and gender as additional predictors in the regression. Using data only from individuals with schizophrenia, separate linear regressions were conducted to determine the relationship between head movement and the PANSS subscale scores in addition to individual items in each of those subscales, with age and gender as confounding variables. The linear regressions were an exploratory analysis to determine the relationship between head movement and individual items on the PANSS, and hence multiple comparisons correction was not conducted. Additionally, a $t$ test was conducted using the average rate of head movement between the individuals with schizophrenia and healthy controls.

Computer Vision-Based Assessment of Motor Functioning in Schizophrenia

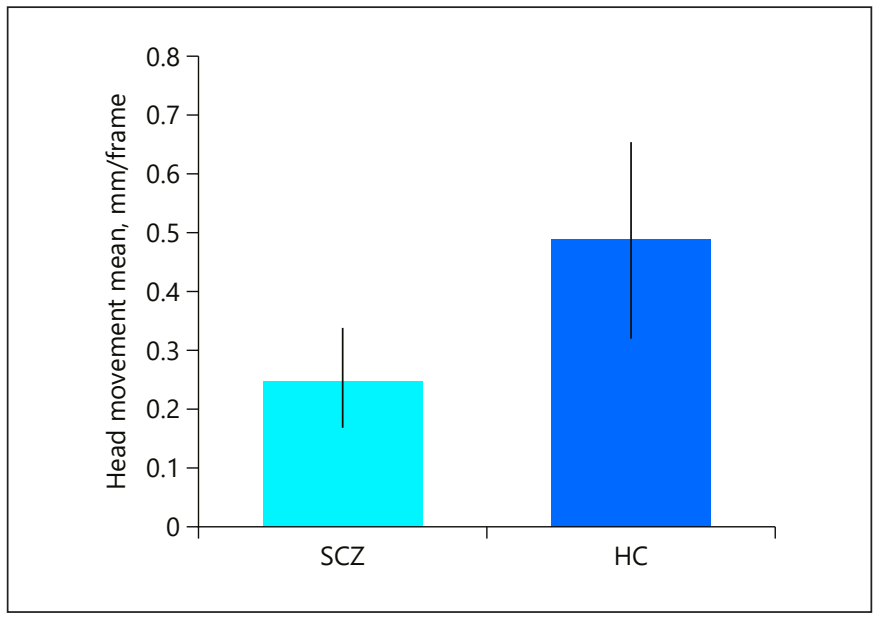

Fig. 1. The rate of head movement in participants with schizophrenia (SCZ) was significantly less than in healthy individuals (HC).

Table 1. Results from multiple logistic regression of head movement, age, and gender with schizophrenia diagnoses

\begin{tabular}{lrlrl}
\hline Variable & Coefficient & SE & \multicolumn{1}{l}{$z$} & $p$ value \\
\hline Constant & -0.456 & 2.051 & -0.222 & 0.824 \\
Head motion & -5.290 & 2.393 & -2.210 & 0.027 \\
Age (years) & 0.074 & 0.046 & 1.611 & 0.107 \\
Gender & -0.416 & 1.030 & -0.404 & 0.686 \\
\hline
\end{tabular}

\section{Data Availability}

The raw video analyzed in this manuscript is considered protected health information. It was not consented for public use and cannot be published. However, the first level of non-identifiable raw abstractions from that data as well as the derivations used to conduct all analyses will be provided alongside the code.

\section{Code Availability}

Code for all methods and analysis presented in this manuscript is publicly available on GitHub (https:/github.com/anzarabbas/ ms_headmov_scz).

\section{Results}

The rate of head movement in participants with schizophrenia ( $1.48 \mathrm{~mm} /$ frame) and those without differed significantly $(2.50 \mathrm{~mm} /$ frame; $p=0.01)$, as is shown in Figure 1 .

\section{Logistic Regression}

Data from 9 healthy controls and 17 individuals with schizophrenia was used for the logistic regressions, 1 in- 
Table 2. Results for the linear regressions conducted with head movement, age, and gender as predictors of PANSS subscale scores

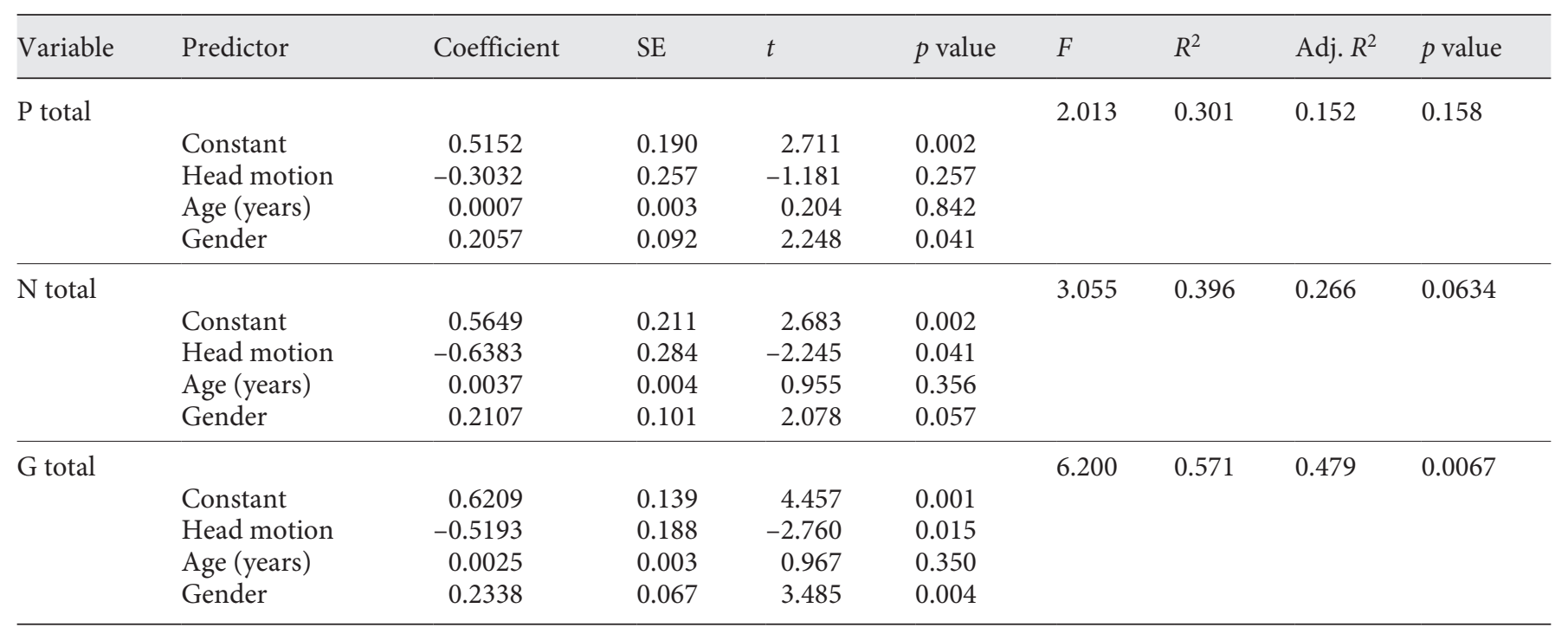

dividual being excluded due to missing PANSS scores. Head movement, age, and gender were able to significantly differentiate healthy controls and individuals with schizophrenia (log likelihood $=-12.13$; Pseudo $r^{2}=0.29$; $p=0.018$ ) with head movement as the only significant predictor (odds ratio $=0.04,95 \%$ CI $0.00-0.48 ; z=-2.21$; $p=0.027$ ) and age demonstrating a marginal effect (odds ratio $=1.07,95 \%$ CI $0.99-1.18 ; z=1.61 ; p=0.1$; Table 1 ).

\section{Linear Regression}

In the data collected from individuals with schizophrenia $(n=18)$, separate linear regressions were conducted with head movement, age, and gender as predictors of each individual item on the PANSS. The results from all of these regressions are detailed in online supplementary Tables 1, 2, and 3 (for all online suppl. material, see www.karger.com/doi/10.1159/000512383). A subset of the results from those regressions are outlined in Tables 2 and 3 .

When correcting for age and gender, head movement had a significant negative association with the $\mathrm{N}$ Total and G Total scores $(p=0.041$ and $p=0.015$, respectively) but not the P Total score ( $p=0.257$; Table 2$)$. Furthermore, when correcting for age and gender, head movement was negatively associated with several individual items on the PANSS (Table 3): blunted affect $(p=0.040)$, emotional withdrawal $(p=0.020)$, poor rapport $(p=$ $0.043)$, motor retardation ( $p=0.004)$, uncooperativeness $(p=0.049)$, poor attention $(p=0.049)$, and lack of judgement and insight $(p=0.003)$.

\section{Discussion}

In the current investigation, we sought to test the hypothesis that motor functioning as measured by the amount of head movement during free behavior, quantified through convolutional neural network-based computer vision models, would serve as a digital marker of schizophrenia symptomatology. We further assessed the ability of digital tools, that is, remote smartphone-based assessments that capture video of individuals' behavior, to measure schizophrenia symptomatology in an accurate, scalable, and objective manner.

The findings demonstrate that head movement during free responses to scripted prompts delivered over a smartphone and recorded through the front-facing camera significantly differentiated healthy controls from individuals with schizophrenia (Table 1). Furthermore, the exploratory results suggest that slowed head movement is a marker of negative symptomatology of schizophrenia, including blunted affect, motor retardation, poor rapport, and emotional withdrawal (Table 2). Additionally, we found preliminary evidence that head movement is associated with uncooperativeness, poor attention, and lack of insight. While the interpretation of this relationship is less straightforward, this may reflect underlying cognitive deficits in schizophrenia associated with negative symptomatology. Our findings align with previously reported motor slowing in individuals with schizophrenia [45-48] and are consistent with the notion that different types of motor symptoms 
Table 3. A subset of the results for the linear regressions conducted with head movement, age, and gender as predictors of individual PANSS items

\begin{tabular}{|c|c|c|c|c|c|c|c|c|c|}
\hline Dependent variable & Predictor & Coefficient & SE & $t$ & $p$ value & $F$ & $R^{2}$ & $\operatorname{Adj} . R^{2}$ & $p$ value \\
\hline Blunted affect & $\begin{array}{l}\text { Constant } \\
\text { Head motion } \\
\text { Age (years) } \\
\text { Gender }\end{array}$ & $\begin{array}{r}0.5832 \\
-0.8133 \\
0.0038 \\
0.2040\end{array}$ & $\begin{array}{l}0.264 \\
0.356 \\
0.005 \\
0.128\end{array}$ & $\begin{array}{r}2.221 \\
-2.284 \\
0.789 \\
1.590\end{array}$ & $\begin{array}{l}0.046 \\
0.040 \\
0.444 \\
0.136\end{array}$ & 2.565 & 0.372 & 0.227 & 0.099 \\
\hline Poor rapport & $\begin{array}{l}\text { Constant } \\
\text { Head motion } \\
\text { Age (years) } \\
\text { Gender }\end{array}$ & $\begin{array}{r}0.6081 \\
-0.8332 \\
-0.0005 \\
0.1107\end{array}$ & $\begin{array}{l}0.275 \\
0.372 \\
0.005 \\
0.134\end{array}$ & $\begin{array}{r}2.208 \\
-2.241 \\
0.100 \\
0.826\end{array}$ & $\begin{array}{l}0.046 \\
0.043 \\
0.922 \\
0.424\end{array}$ & 1.830 & 0.297 & 0.135 & 0.191 \\
\hline Motor retardation & $\begin{array}{l}\text { Constant } \\
\text { Head motion } \\
\text { Age (years) } \\
\text { Gender }\end{array}$ & $\begin{array}{r}0.5972 \\
-1.1118 \\
0.0062 \\
0.0757\end{array}$ & $\begin{array}{l}0.233 \\
0.315 \\
0.004 \\
0.113\end{array}$ & $\begin{array}{r}2.563 \\
-3.534 \\
1.447 \\
0.668\end{array}$ & $\begin{array}{l}0.024 \\
0.004 \\
0.172 \\
0.516\end{array}$ & 4.862 & 0.529 & 0.420 & 0.017 \\
\hline Uncooperativeness & $\begin{array}{l}\text { Constant } \\
\text { Head motion } \\
\text { Age (years) } \\
\text { Gender }\end{array}$ & $\begin{array}{r}0.7059 \\
-0.6860 \\
-0.0019 \\
0.1316\end{array}$ & $\begin{array}{l}0.234 \\
0.315 \\
0.004 \\
0.114\end{array}$ & $\begin{array}{r}3.023 \\
-2.176 \\
-0.438 \\
1.159\end{array}$ & $\begin{array}{l}0.010 \\
0.049 \\
0.669 \\
0.267\end{array}$ & 2.034 & 0.319 & 0.162 & 0.159 \\
\hline Poor attention & $\begin{array}{l}\text { Constant } \\
\text { Head motion } \\
\text { Age (years) } \\
\text { Gender }\end{array}$ & $\begin{array}{r}0.4311 \\
-0.5696 \\
0.0001 \\
0.3322\end{array}$ & $\begin{array}{l}0.194 \\
0.262 \\
0.004 \\
0.094\end{array}$ & $\begin{array}{r}2.224 \\
-2.177 \\
0.031 \\
3.523\end{array}$ & $\begin{array}{l}0.044 \\
0.049 \\
0.976 \\
0.004\end{array}$ & 5.454 & 0.557 & 0.455 & 0.012 \\
\hline
\end{tabular}

may correlate with different aspects of psychotic symptoms [49].

Furthermore, the results demonstrated that motor functioning can be objectively measured using data captured remotely, in the absence of a clinician or a clinical environment, and without the need for any specialized equipment or hardware beyond a smartphone. Video captured of an individual's behavior during remote smartphone-based assessments was sufficient for measurement of head movement as a behavioral symptom of schizophrenia, and may be sufficient for computationally

Computer Vision-Based Assessment of

Motor Functioning in Schizophrenia based measurement of other behavioral symptoms such as changes in facial expressivity, vocal acoustics, and characteristics of speech. With tools emerging that are able to measure all such behavioral symptomatology using computer vision, digital signal processing, and machine learning [34-38, 50-55], these behaviors may be measurable in the same manner that movement behavior was assessed in this investigation.

Abnormalities in motor functioning underlie observable symptomatology in several neurological and neuropsychiatric disorders, including major depressive disor- 
der [56, 57], Parkinson's disease [58], and autism spectrum disorder [59-61], among others. Given the transdiagnostic nature of motor symptomatology, remote and scalable methods for its measurement may have utility for the assessment of treatment response and/or motor side-effects of treatment in both clinical care and clinical research beyond just schizophrenia. With advancements in computationally based measurement of other observable behavioral symptomatology, such as facial expressivity, speech, and physiology, digital measurements could bring immense utility in the monitoring and diagnosis of mental and physical health using remote tools.

It is important to note the key limitations of this study, in particular the multiple comparisons conducted on the modest sample size. With the primary objective of this study being to test the hypothesis that head movement is negatively correlated with schizophrenia diagnosis, the logistic regression provided compelling evidence towards rejection of the null hypothesis. The subsequent linear regressions conducted between head movement and all PANSS items served as a secondary and exploratory aim, allowing for determination of a relationship between head movement and specific schizophrenia symptomatology. With results from the analysis indicating relationships as would be hypothesized based on prior reports, we present them as preliminary findings with the ultimate goal of expanding such an experiment to a wider patient population, allowing for a more fine-grained analysis of head movement as a digital biomarker of schizophrenia symptomatology.

Additionally, the sample size inhibited the ability to age-match the 2 populations, which would have been an important step in alleviating age-related variability in head movement beyond simply correcting for it in the regression. Furthermore, given patients were on a wide range of antipsychotics, it was difficult to assess the effects of individual medications, including antipsychotic-induced motor abnormalities such as tardive dyskinesia, which have the potential to influence head movement measurements. However, involuntary movement behavior would have only increased the head movement intensity observed in the individuals with schizophrenia. Given we were able to reject the null hypothesis, it is possible that antipsychotic-induced head movement did not contribute very strongly as a confounding variable.

Finally, this investigation utilized open-source Python-based software, available to all researchers. As an additional measure, the code that implemented the open- source software for this investigation and subsequent analyses of results have been provided by the authors in the Methods section. This allows for the expansion of the experiment to a wider patient population as mentioned above and the independent validation of the computer vision-based measurement of head movement itself and its implementation in this investigation by other researchers in academic and clinical research, following an open-science framework for the development of digital tools for objective, accurate, and scalable measurement of disease symptomatology in both mental and physical health. Adaptation of such technology will allow for its implementation in larger patient populations, potentially leading to future research that would be able to train machine learning-based classifiers that use head movement along with other quantifiable features to identify the presence and severity of neuropsychiatric disorders such as schizophrenia.

\section{Conclusions}

In this investigation, we demonstrated that head movement measured using computer vision from video captured remotely via smartphones demonstrates validity as a marker of schizophrenia and is a promising metric for negative symptom severity. Use of such technology in clinical care and clinical research settings could allow for accurate measurement of disease symptomatology and treatment response in a scalable and accessible manner, which can support the development of novel treatments for schizophrenia and other mental and physical disorders that involve motor symptomatology.

\section{Acknowledgments}

The authors appreciate the involvement of the clinical, research, and operations staff at both Mount Sinai and AiCure for the development, deployment, and implementation of the technology presented here, and the participants who volunteered to be involved in the research.

\section{Statement of Ethics}

All participants gave their written informed consent for the current study. The study protocol was approved by the Mount Sinai Program for the Protection of Human Subjects and its Institutional Review Board.
Abbas et al. 


\section{Conflict of Interest Statement}

Dr. Mercedes Perez-Rodriguez has received research grant funding from Neurocrine Biosciences Inc., Millennium Pharmaceuticals, Takeda, Merck, and AiCure. She is an advisory board member for Neurocrine Biosciences Inc. and a consultant on an American Foundation for Suicide Prevention (AFSP) grant (LSRG-1-005-16, PI: Baca-Garcia). Anzar Abbas, Vijay Yadav, Vidya Koesmahargyo, Li Zhang, Lei Guan, and Isaac R. GalatzerLevy are employed at AiCure and own shares or are eligible to own shares in AiCure.

\section{Funding Sources}

This study was funded by AiCure LLC (Protocol AICVDX01), GCO: 18-0968-00001-01-PD.

\section{Author Contributions}

A.A., V.Y., V.K., L.Z., L.G., and I.R.G.-L. contributed towards the conception of the study, development of technology used, data analysis conducted, and reporting of the results. E.S., E.R., S.B.R., C.B., P.R., and M.P.-R. carried out all participant recruitment, technology onboarding, and clinical data collection.

\section{References}

1 Brekke JS, Raine A, Ansel M, Lencz T, Bird L. Neuropsychological and psychophysiological correlates of psychosocial functioning in schizophrenia. Schizophr Bull. 1997;23(1): $19-28$

2 Ellegood J, Henkelman RM, Lerch JP. Neuroanatomical Assessment of the Integrin $\beta 3$ Mouse Model Related to Autism and the Serotonin System Using High Resolution MRI. Front Psychiatry. 2012 Apr;3:37.

3 Morrens M, Docx L, Walther S. Beyond boundaries: in search of an integrative view on motor symptoms in schizophrenia. Front Psychiatry. 2014 Oct;5:145.

4 Sullivan EV, Shear PK, Lim KO, Zipursky RB, Pfefferbaum A. Cognitive and motor impairments are related to gray matter volume deficits in schizophrenia. Biol Psychiatry. 1996 Feb;39(4):234-40.

5 Sullivan EV, Shear PK, Zipursky RB, Sagar HJ, Pfefferbaum A. A deficit profile of executive, memory, and motor functions in schizophrenia. Biol Psychiatry. 1994 Nov;36(10):641-53.

6 Wolff AL, O'Driscoll GA. Motor deficits and schizophrenia: the evidence from neuroleptic-naïve patients and populations at risk. J Psychiatry Neurosci. 1999 Sep;24(4):304-14.

7 Brébion G, Amador X, Smith M, Malaspina D, Sharif Z, Gorman JM. Depression, psychomotor retardation, negative symptoms, and memory in schizophrenia. Neuropsychiatry Neuropsychol Behav Neurol. 2000 Jul;13(3): $177-83$.

8 Winograd-Gurvich C, Fitzgerald PB, Georgiou-Karistianis N, Bradshaw JL, White OB. Negative symptoms: A review of schizophrenia, melancholic depression and Parkinson's disease. Brain Res Bull. 2006 Oct;70(4-6) $312-21$.

9 Maes M, Sirivichayakul S, Kanchanatawan B, Carvalho AF. In schizophrenia, psychomotor retardation is associated with executive and memory impairments, negative and psychotic symptoms, neurotoxic immune products and lower natural IgM to malondialdehyde. World J Biol Psychiatry. 2020 Jun;21(5):383401.
10 Kay SR, Opler LA. L-dopa in the treatment of negative schizophrenic symptoms: a singlesubject experimental study. Int J Psychiatry Med. 1985-1986;15(3):293-8.

11 Bervoets C, Docx L, Sabbe B, Vermeylen S, Van Den Bossche MJ, Morsel A, et al. The nature of the relationship of psychomotor slowing with negative symptomatology in schizophrenia. Cogn Neuropsychiatry. 2014;19(1):36-46.

12 Blanchard JJ, Kring AM, Horan WP, Gur R. Toward the next generation of negative symptom assessments: the collaboration to advance negative symptom assessment in schizophrenia. Schizophr Bull. 2011 Mar; 37(2):291-9.

13 Kay SR. Positive-negative symptom assessment in schizophrenia: psychometric issues and scale comparison. Psychiatr Q. 1990; 61(3):163-78

14 Wilk CM, Gold JM, Humber K, Dickerson F, Fenton WS, Buchanan RW. Brief cognitive assessment in schizophrenia: normative data for the Repeatable Battery for the Assessment of Neuropsychological Status. Schizophr Res. 2004 Oct; $70(2-3): 175-86$.

15 Jahn T, Cohen R, Hubmann W, Mohr F, Köhler I, Schlenker R, et al. The Brief Motor Scale (BMS) for the assessment of motor soft signs in schizophrenic psychoses and other psychiatric disorders. Psychiatry Res. 2006 Jun;142(2-3):177-89.

16 Minassian A, Henry BL, Geyer MA, Paulus MP, Young JW, Perry W. The quantitative assessment of motor activity in mania and schizophrenia. J Affect Disord. 2010 Jan; 120(1-3):200-6.

17 Walther S, Strik W. Motor symptoms and schizophrenia. Neuropsychobiology. 2012; 66(2):77-92.

18 Erhart SM, Marder SR, Carpenter WT. Treatment of schizophrenia negative symptoms: future prospects. Schizophr Bull. 2006 Apr; 32(2):234-7.

19 Fusar-Poli P, Papanastasiou E, Stahl D, Rocchetti M, Carpenter W, Shergill S, et al. Treatments of Negative Symptoms in Schizophrenia: Meta-Analysis of 168 Randomized Placebo-Controlled Trials. Schizophr Bull. 2015 Jul;41(4):892-9.
20 Millan MJ, Fone K, Steckler T, Horan WP Negative symptoms of schizophrenia: clinical characteristics, pathophysiological substrates, experimental models and prospects for improved treatment. Eur Neuropsychopharmacol. 2014 May;24(5):645-92.

21 Singh SP, Singh V, Kar N, Chan K. Efficacy of antidepressants in treating the negative symptoms of chronic schizophrenia: meta-analysis. Br J Psychiatry. 2010 Sep;197(3):174-9.

22 Kirkpatrick B, Fenton WS, Carpenter WT Jr, Marder SR. The NIMH-MATRICS consensus statement on negative symptoms. Schizophr Bull. 2006 Apr;32(2):214-9.

23 King DJ. Drug treatment of the negative symptoms of schizophrenia. Eur Neuropsychopharmacol. 1998 Feb;8(1):33-42.

24 Möller HJ. Clinical evaluation of negative symptoms in schizophrenia. Eur Psychiatry. 2007 Sep;22(6):380-6.

25 Prikryl R, Kasparek T, Skotakova S, Ustohal L, Kucerova H, Ceskova E. Treatment of negative symptoms of schizophrenia using repetitive transcranial magnetic stimulation in a double-blind, randomized controlled study. Schizophr Res. 2007 Sep;95(1-3):151-7.

26 Walther S, Koschorke P, Horn H, Strik W. Objectively measured motor activity in schizophrenia challenges the validity of expert ratings. Psychiatry Res. 2009 Oct;169(3):187-90.

27 Brown KW, White T, Palmer D. Movement disorders and psychological tests of frontal lobe function in schizophrenic patients. Psychol Med. 1992 Feb;22(1):69-77.

28 Manschreck TC, Maher BA, Rucklos ME, Vereen DR Jr, Ader DN. Deficient motor synchrony in schizophrenia. J Abnorm Psychol. 1981 Aug;90(4):321-8.

29 Manschreck TC, Maher BA, Waller NG, Ames D, Latham CA. Deficient motor synchrony in schizophrenic disorders: clinical correlates. Biol Psychiatry. 1985 Sep;20(9):990-1002.

30 Steffy R, Waldman I. Schizophrenics' reaction time: north star or shooting star? In: Cromwell R, Snyder C, editors. Schizophrenia origins, processes, treatment, and outcome. New York (NY): Oxford University Press; 1993. pp. 111-34.
Computer Vision-Based Assessment of Motor Functioning in Schizophrenia
Digit Biomark 2021;5:29-36 DOI: $10.1159 / 000512383$ 
31 Vrtunski PB, Simpson DM, Meltzer HY. Voluntary movement dysfunction in schizophrenics. Biol Psychiatry. 1989 Mar;25(5): 529-39.

32 Walther S, Horn H, Razavi N, Koschorke P, Müller TJ, Strik W. Quantitative motor activity differentiates schizophrenia subtypes. Neuropsychobiology. 2009;60(2):80-6.

33 Insel TR. Digital phenotyping: a global tool for psychiatry. World Psychiatry. 2018 Oct; 17(3):276-7.

34 Alvino C, Kohler C, Barrett F, Gur RE, Gur RC, Verma R. Computerized measurement of facial expression of emotions in schizophrenia. J Neurosci Methods. 2007 Jul;163(2): 350-61.

35 Dudek A, Wilczyński KM, Krysta K, Pęszor D, Martyniak E, Wojciechowska M, et al. Analysis of facial expressions in patients with schziophrenia, in comparison with a healthy control - case study. Psychiatr Danub. 2017 Sep;29(Suppl 3):584-9.

36 Gaebel W, Wölwer W. Facial expressivity in the course of schizophrenia and depression. Eur Arch Psychiatry Clin Neurosci. 2004 Oct; 254(5):335-42.

37 Jian B-L, Chen C-L, Chu W-L, Huang M-W. The facial expression of schizophrenic patients applied with infrared thermal facial image sequence. BMC Psychiatry. 2017;17(1):229.

38 Thevenot J, Lopez MB, Hadid A. A survey on computer vision for assistive medical diagnosis from faces. IEEE J Biomed Health Inform. 2018 Sep;22(5):1497-511.

39 Murphy-Chutorian E, Trivedi MM. Head pose estimation in computer vision: a survey. IEEE Trans Pattern Anal Mach Intell. 2009 Apr;31(4):607-26.

40 Alghowinem S, Goecke R, Wagner M, Parkerx G, Breakspear M. Head pose and movement analysis as an indicator of depression. Proceedings of the 2013 Humaine Association Conference on Affective Computing and Intelligent Interaction [Internet]. USA: IEEE Computer Society; 2013. p. 283-288. (ACII '13).

41 Opler MG, Yavorsky C, Daniel DG. Positive and Negative Syndrome Scale (PANSS) Training: Challenges, Solutions, and Future Directions. Innov Clin Neurosci. 2017 Dec; 14(11-12):77-81.
42 Baltrusaitis T, Robinson P, Morency LP. OpenFace: An open source facial behavior analysis toolkit. In: 2016 IEEE Winter Conference on Applications of Computer Vision (WACV). Lake Placid, NY, USA: IEEE; 2016. pp. 1-10. Available from http://ieeexplore. ieee.org/document/74775553/.

43 Amos B, Ludwiczuk B, Satyanarayanan M OpenFace: A general-purpose face recognition library with mobile applications. Available from: http://http://cmusatyalab.github. io/openface/.

44 Baltrusaitis T, Zadeh A, Lim YC, Morency LP. OpenFace 2.0: Facial Behavior Analysis Toolkit. In: 2018 13th IEEE International Conference on Automatic Face Gesture Recognition (FG 2018). 2018. p. 59-66.

45 Jogems-Kosterman BJ, Zitman FG, Van Hoof JJ, Hulstijn W. Psychomotor slowing and planning deficits in schizophrenia. Schizophr Res. 2001 Mar;48(2-3):317-33.

46 van Hoof JJ, Jogems-Kosterman BJ, Sabbe BG, Zitman FG, Hulstijn W. Differentiation of cognitive and motor slowing in the Digit Symbol Test (DST): differences between depression and schizophrenia. J Psychiatr Res. 1998 Mar-Apr;32(2):99-103.

47 Morrens M, Hulstijn W, Sabbe B. Psychomotor slowing in schizophrenia. Schizophr Bull. 2007 Jul;33(4):1038-53.

48 Morrens M, Hulstijn W, Matton C, Madani Y, van Bouwel L, Peuskens J, et al. Delineating psychomotor slowing from reduced processing speed in schizophrenia. Cogn Neuropsychiatry. 2008 Nov;13(6):457-71.

49 Wang SM, Ouyang WC, Wu MY, Kuo LC. Relationship between motor function and psychotic symptomatology in young-adult patients with schizophrenia. Eur Arch Psychiatry Clin Neurosci. 2020 Apr;270(3):37382.

50 Javitt DC, Sweet RA. Auditory dysfunction in schizophrenia: integrating clinical and basic features. Nat Rev Neurosci. 2015 Sep;16(9): 535-50.

51 Kayi ES, Diab M, Pauselli L, Compton M, Coppersmith G. Predictive Linguistic Features of Schizophrenia. ArXiv181009377 Cs [Internet]. 2018 Oct 22 [cited May 29, 2020]; Available from: http://arxiv.org/abs/1810.09377.
52 Kliper R, Vaizman Y, Weinshall D, Portuguese $\mathrm{S}$. Evidence for depression and schizophrenia in speech prosody. In 2019 [cited May 29, 2020]. p. 85-8. Available from: https://exlingsociety.com/images/Proceedings/ExLing_2010/03_0022_000142.pdf.

53 Martínez-Sánchez F, Muela-Martínez JA, Cortés-Soto P, García Meilán JJ, Vera Ferrándiz JA, Egea Caparrós A, et al. Can the Acoustic Analysis of Expressive Prosody Discriminate Schizophrenia? Span J Psychol. 2015 Nov; 18:E86.

54 Parola A, Simonsen A, Bliksted V, Fusaroli R. Voice patterns in schizophrenia: a systematic review and Bayesian meta-analysis. Schizophr Res. 2020 Feb;216:24-40.

55 Tahir Y, Yang Z, Chakraborty D, Thalmann N, Thalmann D, Maniam Y, et al. Non-verbal speech cues as objective measures for negative symptoms in patients with schizophrenia. PLoS One. 2019 Apr;14(4):e0214314.

56 Caligiuri MP, Ellwanger J. Motor and cognitive aspects of motor retardation in depression. J Affect Disord. 2000 Jan-Mar;57(1-3): 83-93.

57 Szabadi E, Bradshaw CM, Besson JA. Elongation of pause-time in speech: a simple, objective measure of motor retardation in depression. Br J Psychiatry. 1976 Dec;129(6):592-7.

58 Sveinbjornsdottir S. The clinical symptoms of Parkinson's disease. J Neurochem. 2016 Oct; 139 Suppl 1:318-24.

59 Bhat AN, Landa RJ, Galloway JC. Current perspectives on motor functioning in infants, children, and adults with autism spectrum disorders. Phys Ther. 2011 Jul;91(7):1116-29.

60 Rinehart NJ, Bellgrove MA, Tonge BJ, Brereton AV, Howells-Rankin D, Bradshaw JL. An examination of movement kinematics in young people with high-functioning autism and Asperger's disorder: further evidence for a motor planning deficit. J Autism Dev Disord. 2006 Aug;36(6):757-67.

61 Provost B, Lopez BR, Heimerl S. A comparison of motor delays in young children: autism spectrum disorder, developmental delay, and developmental concerns. J Autism Dev Disord. 2007 Feb;37(2):321-8. 